\title{
Associações de aves com insetos sociais: um sumário no Sudeste do Brasil
}

\author{
Ivan Sazima' \& Giulia B. D'Angelo²
}

\begin{abstract}
1. Museu de Zoologia, Universidade Estadual de Campinas, Caixa Postal 6109, 13083-970, Campinas, SP, Brasil. (isazima@gmail.com) 2. Instituto de Biologia, Caixa Postal 6109, Universidade Estadual de Campinas, 13083-970, Campinas, SP, Brasil.
\end{abstract}

\begin{abstract}
Association of birds with social insects: a summary in Southeastern Brazil. Birds may aggregate with various kinds of social insects (ants, wasps, termites). These associations may be grouped in three types, one of them related to feeding activity and the other two, to breeding activity: 1) foraging with army ants; 2) nesting close to active wasp or ant colonies; 3 ) nesting in active termitaria. These three association types are examples of commensalism. Herein we summarise associations of birds with social insects that we recorded in South-eastern Brazil. Following army ants was usual to rare for a great variety of bird species, from the Accipitridae to Tyrannidae. Nesting close to active wasp colonies was usual for a species of the Rhynchocyclidae. Nesting in active arboreal termitaria was usual for two species of the Trogonidae and rare for two species of the Psittacidae. Nesting in active ground termitaria was rare for a species of the Picidae and a species of the Strigidae. However, regional qualitative and quantitative variations probably occur, which would merit this study type across habitats and regions distinct from those we studied. Natural history-oriented studies will likely disclose a greater species richness and variety of birds that associate with active colonies of social insects.
\end{abstract}

KEYWORDS. Foraging, army ants, nesting, wasp colonies, termitaria.

RESUMO. As aves podem se agregar a diversos tipos de insetos sociais (formigas, vespas, cupins). Estas associações podem ser agrupadas em três tipos, um deles relacionado a atividade alimentar e dois, a atividade reprodutiva: 1) forragear seguindo formigas de correição; 2) nidificar nas proximidades de colônias ativas de vespas e formigas; 3 ) nidificar em colônia ativas de cupins. Os três tipos de associação podem ser considerados como exemplos de comensalismo. Sumariamos aqui as associações de aves com insetos sociais que observamos no Sudeste do Brasil. Seguir formigas de correição foi habitual a raro para uma grande variedade de espécies, de Accipitridae a Tyrannidae. Nidificar nas proximidades de colônias ativas de vespas foi habitual para uma espécie de Rhynchocyclidae. Nidificar em cupinzeiros arborícolas ativos foi habitual para duas espécies de Trogonidae e ocasional para duas espécies de Psittacidae. Nidificar em cupinzeiros terrestres ativos foi raro para uma espécie de Picidae e uma espécie de Strigidae. Entretanto, variações quali e quantitativas regionais provavelmente ocorrem, o que justificaria este tipo de estudo em habitats e regiões distintas das aqui estudadas. Estudos orientados para história natural deverão revelar maior riqueza de espécies e variedade de aves que se associam a colônias ativas de insetos sociais.

PALAVRAS-CHAVE. Forrageio, formigas-correição, nidificação, colônias de vespas, cupinzeiros.

As aves podem se agregar a diversos tipos de insetos sociais, como formigas, vespas e cupins (MYERs, 1935; BrightSMith, 2000; WinKLeR \& Christie, 2002; Willis \& ONIKI, 2003; QuinN \& UeTA, 2008). A maioria destas associações de aves com insetos pode ser convenientemente agrupada em três tipos, um deles relacionado a atividade alimentar e dois, a atividade reprodutiva: 1) forragear seguindo formigas de correição; 2) nidificar nas proximidades de colônias ativas de vespas ou formigas; 3) nidificar em colônias ativas de cupins. Estes três tipos de associação podem ser considerados como exemplos de comensalismo.

Seguir formigas de correição é uma associação das mais estudadas, ocorrendo nas regiões tropicais das Américas e da África (WiLlis \& ONIKI, 2003, 2008). Nos Neotrópicos, as aves acompanham as colunas de caça de formigas dos gêneros Eciton Latreille, 1804 e Labidus Jurine, 1807 (Swartz, 2001; Willis \& ONIKI, 2008; Pizo $\&$ Melo, 2010). Durante esta atividade, as aves apanham pequenos animais (principalmente invertebrados) que procuram escapar das formigas caçadoras (Sick, 1997; WILLIS \& ONIKI, 2003).

Nidificar próximo a ninhos ativos de vespas e formigas é considerado como uma forma de proteção à prole (Myers, 1935; Joyce, 1993; Quinn \& Ueta, 2008), ocorrendo em diversas espécies de aves. Na Região Neotropical, a associação mais bem conhecida é a de aves do gênero Cacicus Lacépède, 1799 (Icteridae), além de Tolmomyias Hellmayr, 1927 (Rhynchocyclidae), com vespas coloniais de Polistinae (MYers, 1935; ROBINSON, 1985; FitzPatrick, 2004; Cazal et al., 2009; Fraga, 2011; Menezes et al., 2014).

Diversas aves Psittacidae, Trogonidae, Alcedinidae e Picidae, que escavam ninhos em troncos ou barrancos, usam colônias de cupins para nidificar (MYERs, 1935; Collar, 1997, 2001; Sick, 1997; BrightSMith, 2000; WinKLeR \& Christie, 2002). Nos Neotrópicos, as aves mais conhecidas que usam colônias ativas de cupins são surucuás do gênero Trogon Lesson, 1828 e periquitos de Brotogeris Vigors, 1825 e Eupsittula Bonaparte, 1853 (Sick, 1997; Brightsmith, 2000; Paranhos et al., 2008). Embora haja publicações de qualidade sobre estes três tipos de associação no Brasil, não encontramos trabalho que tratasse do conjunto de associações de aves com insetos sociais de modo abrangente. Por este motivo, sumariamos aqui as associações de aves com insetos sociais que observamos ao longo de nove anos de estudos sobre história natural de aves no Sudeste do Brasil (estados de 
São Paulo e Rio de Janeiro). Adicionalmente, comentamos sobre as circunstâncias nas quais essas associações ocorrem e a sua relação com o modo de vida das aves e dos insetos envolvidos.

\section{MATERIAL E MÉTODOS}

As associações aqui relatadas e documentadas foram registradas durante estudos sobre história natural de aves nas regiões de Águas de São Pedro, Campinas, Ilhabela e Ubatuba (estado de São Paulo), Itatiaia e ParatiMirim (estado do Rio Janeiro), entre novembro de 2005 a dezembro de 2014. Nas regiões de Águas de São Pedro e Campinas, a fitofisionomia predominante é Cerrado, porém há trechos com Mata Semidecídua; nas regiões de Itatiaia, Parati-Mirim, Ubatuba e Ilhabela, a fitofisionomia é Mata Atlântica (EITEN, 1970, 1992).

Durante os estudos em campo, examinamos colunas de caça de formigas de correição (Ecitoninae) e registramos as aves acompanhantes. Registramos ninhos de aves que escavam troncos, barrancos e cupinzeiros, para nidificar e examinamos colônias ativas de cupins arborícolas (Nasutitermitinae) e terrícolas (Syntermitinae), registrando ninhos de aves aí escavados. Examinamos colônias de vespas eussociais (Polistinae) e registramos os ninhos de aves construídos em ramos próximos (até $1,5 \mathrm{~m})$ e, também, os ninhos das mesmas espécies de aves construídos em ramos que não contivessem colônias de vespas. Identificamos e fotografamos as espécies de aves e as colônias de insetos (para algumas espécies de cupins, nível de gênero somente). Consideramos como habitual a associação cujas frequências de ocorrências fossem $>50 \%$; ocasional, $30-50 \%$; rara, $<30 \%$. Fotografias digitais, representativas das associações estudadas, estão depositadas no acervo de imagens do Museu de Zoologia da Universidade Estadual de Campinas (ZUEC).

Os nomes de autores e datas da descrição original para as espécies que constam das tabelas seguem aqui. Aves: Rupornis magnirostris (Gmelin, 1789), Crotophaga ani Linnaeus 1758, Piaya cayana (Linnaeus, 1766), Athene cunicularia (Molina, 1782), Trogon surrucura Vieillot, 1817, Trogon viridis Linnaeus, 1766, Colaptes campestris (Vieillot, 1818), Brotogeris chiriri (Vieillot, 1818), Brotogeris tirica (Gmelin, 1788), Dendrocincla turdina (Lichtenstein, 1820), Dendrocolaptes platyrostris Spix, 1825, Phacellodomus erythrophtalmus (Wied, 1821), Myiobius barbatus (Gmelin, 1789), Myiothlypis rivularis (Wied, 1821), Tolmomyias sulphurescens (Spix, 1825), Mackenziaena severa (Lichtenstein, 1823), Pyriglena leucoptera (Vieillot, 1818), Thamnophilus palliatus (Lichtenstein, 1822), Lanio melanops (Vieillot, 1818), Tachyphonus coronatus (Lichtenstein, 1822), Ramphocelus bresilius (Linnaeus, 1766), Myiozetetes similis (Spix, 1825). Insetos: Cornitermes cf. cumulans (Kollar, 1832), Cornitermes cf. silvestrii Emerson, 1949, Apoica pallens (Fabricius, 1804), Pseudopolybia vespiceps (de Saussure, 1863), Eciton burchellii Westwood, 1842, Labidus praedator (Smith, 1858).

\section{RESULTADOS E DISCUSSÃO}

Observamos que seguir formigas de correição foi habitual a raro para uma grande variedade de espécies, de Accipitridae a Tyrannidae (Tab. I). Registramos aves seguidoras das colunas de caça de formigas de correição tanto dentro da mata como na borda (Figs 1,2). Dentro da mata, as colunas eram seguidas principalmente por espécies de Dendrocolaptidae e Thamnophilidae (Tab. I), aves que também ficavam próximas da borda, porém ao abrigo da vegetação. Quando as colunas de formigas passavam principalmente por uma área aberta, atraíam a atenção de espécies de Accipitridae, Cuculidae e Parulidae, além de Thraupidae (Tab. I) que forrageavam fora da mata ou na sua borda.

Observamos que a frequência de uma determinada espécie de ave junto às colunas de caça das formigas depende de circunstâncias diversas, incluindo a sua presença no momento de passagem das formigas e a sua frequência local. Por exemplo, as espécies que registramos com maior frequência (Tab. I) são comuns no litoral norte do estado de São Paulo (observ. pess.). O maior número de espécies de aves que observamos associadas às formigas foi oito, seguindo Labidus praedator em borda de mata, ambiente no qual as aves são mais fáceis de visualizar que dentro da mata. $\mathrm{O}$ menor número foi apenas uma ave associada a esta mesma espécie de formiga (entretanto, também registramos apenas uma espécie de ave associada a Eciton burchellii). Ambas as espécies de formigas são seguidas por aves, embora $L$. praedator pareça oferecer mais dificuldade às seguidoras devido ao fato das suas colunas de caça saírem principalmente à noite (WILLIS \& ONIKI, 2008).

As aves seguidoras Dendrocincla turdina, Mackenziaena severa e Pyriglena leucoptera são insetívoras que seguem correições habitualmente (WILlis \& ONIKI 1978, 2003; SiCK, 1997; MARANTZ et al., 2003; ZiMMER \& IsLER, 2003; PARRINI, 2015) e que foram frequentes nos nossos registros (Tab. I). Por outro lado, Tachyphonus coronatus é uma ave onívora (Sick, 1997; HiLty, 2011) que também foi frequente nos nossos registos (Tab. I). Portanto, dieta insetívora não parece ser condição necessária para uma determinada espécie de ave ser ou não seguidora de formigas.

A associação de aves seguidoras de formigas de correição foi relatada em numerosos estudos feitos por E. O. Willis e Y. Oniki e está sumariada em três artigos principais (WILLIS \& ONIKI, 1978, 2003, 2008). Entretanto, estudos adicionais ainda podem trazer novidades sobre $\mathrm{o}$ tema, e.g., aves seguidoras apresando formigas de correição, interações competitivas entre aves seguidoras, ou aves de rapina seguidoras apresando outras espécies de aves seguidoras (Stotz, 1992; Pizo \& Melo, 2010; SAzima, 2010).

Observamos que nidificar nas proximidades de 
Tab. I. Associações de aves com Hymenoptera (formigas e vespas), observadas no Sudeste de Brasil. A classificação das aves segue o CBRO (2014), mas famílias, gêneros e espécies em ordem alfabética. Para formigas, frequência de ocorrência expressa em número de vezes que uma espécie de ave foi observada junto à correição, dividida pelo número de correições registradas. Para vespas, número de vezes em que ninhos da ave foram observados junto à colônia de vespas, dividido pelo número de vezes em que ninhos da ave foram encontrados. Números entre parênteses são frequência de ocorrência em porcentagem (\%): habitual $(>50)$, ocasional $(30-50)$, raro $(<30)$.

\begin{tabular}{|c|c|c|c|}
\hline Espécies de aves & Espécies de insetos & Tipo de associação & Ocorrência \\
\hline ACCIPITRIDAE & FORMICIDAE & & \\
\hline Rupornis magnirostris & Eciton burchellii & Seguir correição & $2: 7(28,5)$ \\
\hline \multicolumn{4}{|l|}{ CUCULIDAE } \\
\hline Crotophaga ani & Labidus praedator & Seguir correição & $1: 6(16,6)$ \\
\hline Piaya cayana & Labidus praedator & Seguir correição & $1: 6(16,6)$ \\
\hline \multicolumn{4}{|l|}{ DENDROCOLAPTIDAE } \\
\hline \multirow[t]{2}{*}{ Dendrocincla turdina } & Eciton burchellii & Seguir correição & $6: 7(85,7)$ \\
\hline & Labidus praedator & Seguir correição & $5: 6(83,3)$ \\
\hline Dendrocolaptes platyrostris & Eciton burchellii & Seguir correição & $2: 7(28,6)$ \\
\hline \multicolumn{4}{|l|}{ FURNARIIDAE } \\
\hline Phacellodomus erythrophtalmus & Labidus praedator & Seguir correição & $2: 6(33,3)$ \\
\hline \multicolumn{4}{|l|}{ ONYCHORHYNCHIDAE } \\
\hline Myiobius barbatus & Labidus praedator & Seguir correição & $1: 6(16,6)$ \\
\hline \multicolumn{4}{|l|}{ PARULIDAE } \\
\hline Myiothlypis rivularis & Labidus praedator & Seguir correição & $4: 6(66,6)$ \\
\hline RHYNCHOCYCLIDAE & VESPIDAE & & \\
\hline \multirow[t]{2}{*}{ Tolmomyias sulphurescens } & Apoica pallens & Ninho próximo à colônia & $4: 7(57,1)$ \\
\hline & Pseudopolybia vespiceps & Ninho próximo à colônia & $1: 7(14,3)$ \\
\hline \multicolumn{4}{|l|}{ THAMNOPHILIDAE } \\
\hline Mackenziaena severa & Labidus praedator & Seguir correição & $4: 6(66,6)$ \\
\hline \multirow[t]{2}{*}{ Pyriglena leucoptera } & Eciton burchellii & Seguir correição & $6: 7(85,7)$ \\
\hline & Labidus praedator & Seguir correição & $5: 6(83,3)$ \\
\hline Thamnophilus palliatus & Labidus praedator & Seguir correição & $2: 6(33,3)$ \\
\hline \multicolumn{4}{|l|}{ THRAUPIDAE } \\
\hline Lanio melanops & Eciton burchellii & Seguir correição & $2: 7(28,5)$ \\
\hline Ramphocelus bresilius & Eciton burchellii & Seguir correição & $4: 7(57,1)$ \\
\hline Tachyphonus coronatus & Labidus praedator & Seguir correição & $4: 6(66,6)$ \\
\hline \multicolumn{4}{|l|}{ TYRANNIDAE } \\
\hline Myiozetetes similis & Labidus praedator & Seguir correição & $1: 6(16,6)$ \\
\hline
\end{tabular}

colônias ativas de vespas foi habitual para uma espécie de Rhynchocyclidae (Tab. I). Registramos apenas Tolmomyias sulphurescens, que nidificou próximo a ninhos de vespas Polistinae (Figs 3, 4). Dos cinco ninhos da ave encontrados em associação com vespas, em quatro casos a vespa era Apoica pallens, considerada agressiva na defesa das suas colônias (MYers, 1935; SomaviLla et al., 2012). Recentemente, ninhos de T. sulphurescens foram registrados próximos a cinco espécies de vespas eussociais e uma de abelha, no Sudeste do Brasil (Menezes et al., 2014). Em certas regiões, a maioria dos ninhos de T. sulphurescens é construída na vizinhança de colônias de vespas agressivas (FitzPATRICK, 2004). Algumas espécies de Tolmomyias constroem habitualmente seus ninhos próximos a colônias de vespas (HAVERSCHMIDT, 1968), incluindo as agressivas dos gêneros Chartergus Lepeletier, 1836 e Polybia Latreille, 1802 (MYERS, 1935; FitZPATRICK, 2004). No Nordeste do Brasil, Tolmomyias flaviventris (Wied, 1831) constrói seus ninhos na proximidade de colônias de vespas e de formigas (CAZAL et al., 2009).

A construção de ninhos por algumas espécies de aves nas proximidades de colônias ativas de vespas é considerada protetiva contra ataques de predadores, como mamíferos arborícolas, aves de rapina e serpentes (MYERS, 1935; Robinson, 1985; JoYCE, 1993; FitZPATRICK, 2004; CAzal et al., 2009; Somavilla et al., 2013). Primatas que se aproximam dos ninhos do icterídeo Cacicus cela
(Linnaeus, 1758) são repelidos por ataques de vespas Polistinae, na América Central (RobInson, 1985). Maior sucesso reprodutivo de Campylorhynchus rufinucha (Lesson, 1838) e defesa de seus ninhos contra primatas, quando próximo a colônias de vespas Polistinae, foi elegantemente demonstrado por JoYCE (1993), também na América Central. Porém, serpentes alcançam ninhegos dentro dos ninhos pêndulos de Tolmomyias sulphurescens (SILVA, 2014), talvez porque este tipo de predador não perturbe, com seu deslocamento pelos ramos, as colônias de vespas que estiverem nas proximidades. Portanto, ainda que as vespas ofereçam certo grau de proteção às aves que nidificam na proximidade das suas colônias (RoBINSON, 1985; JoYCE, 1993), alguns tipos de predadores superam os mecanismos defensivos de suas presas potenciais ou efetivas (Goodenough et al., 2010).

Observamos que nidificar em cupinzeiros arborícolas ativos foi habitual para duas espécies de Trogonidae e raro para duas espécies de Psittacidae (Tab. II). Quando as aves escavam seu ninho, os cupins consertam o dano ao mesmo tempo, assim separando o ninho das aves do resto do cupinzeiro (Collar, 2001; IS, observ. pess.). Diversas espécies de Trogonidae e Psittacidae comem alguns cupins durante a escavação do ninho (SAZIMA, 1989; Collar, 2001) (Figs. 5, 6). Os nossos resultados estão de acordo com o que é conhecido para o gênero Trogon, que escava habitualmente seu ninho em cupinzeiros 

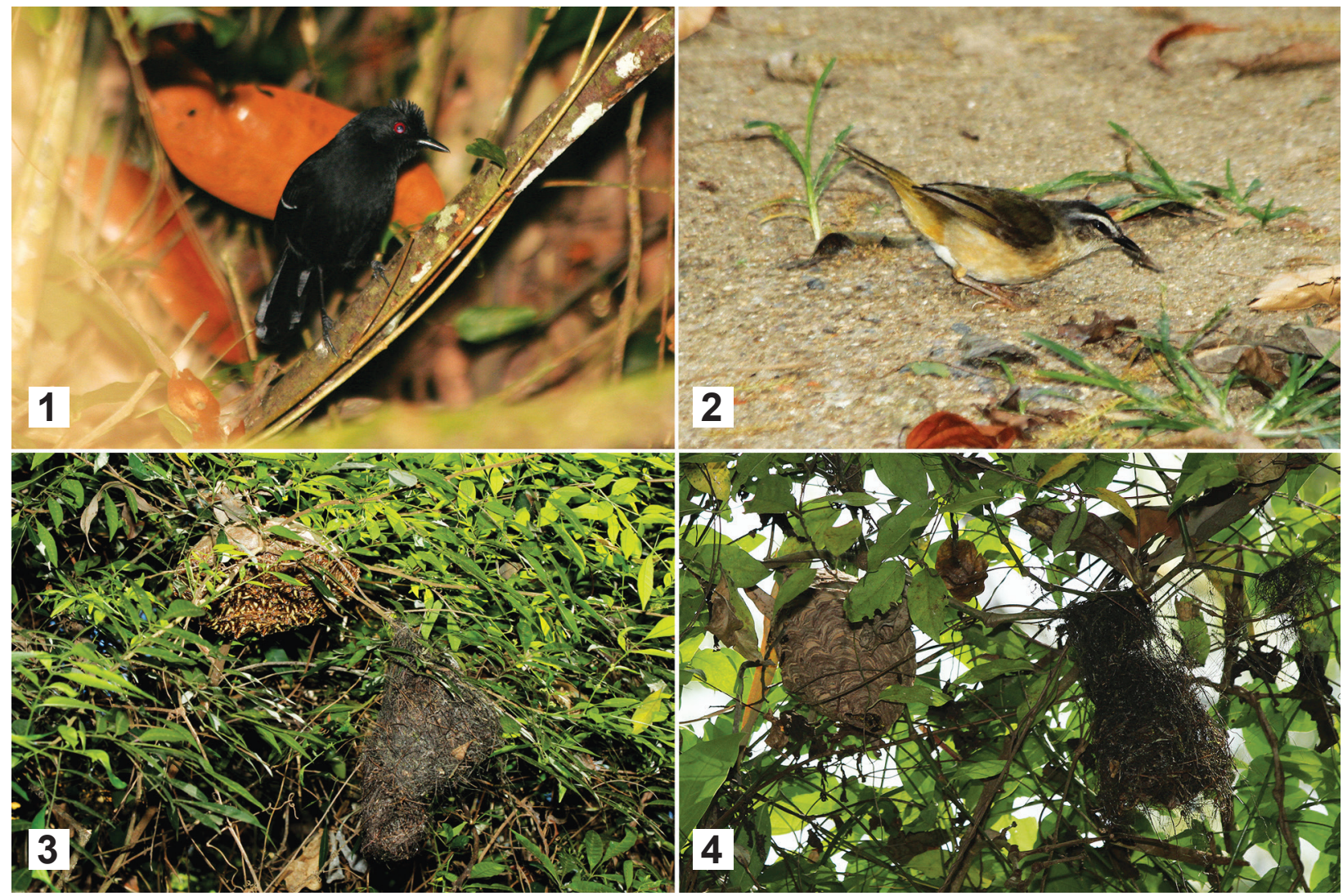

Figs 1-4. Associações de aves com Hymenoptera: 1, no interior de mata, um macho de papa-taoca-do-sul (Pyriglena leucoptera) espreita presas potenciais afugentadas pela atividade das formigas de correição Eciton burchellii; 2, na margem de mata, um pula-pula-ribeirinho (Myiothlypis rivularis) lida com inseto afugentado pelas formigas de correição Labidus praedator; 3, um ninho do bico-chato-de-orelha-preta (Tolmomyias sulphurescens) construído próximo a uma colônia ativa do marimbondo-chapéu (Apoica pallens); 4, outro ninho da mesma espécie de ave, construído próximo a uma colônia ativa do marimbondo-da-mata (Pseudopolybia vespiceps). Créditos: Ivan Sazima (1-4).

Tab. II. Associações de aves com Isoptera (cupins), observadas no Sudeste de Brasil. A classificação das aves segue o CBRO (2014), mas famílias, gêneros e espécies estão em ordem alfabética. Frequência de ocorrência expressa em número de vezes que o ninho de uma espécie de ave foi observado, dividida pelo número de vezes em que o ninho da ave foi registrado em colônia de cupins. Números entre parênteses são frequência de ocorrência em porcentagem (\%): habitual ( $>50)$, ocasional $(30-50)$, raro $(<30)$.

\begin{tabular}{lccc}
\hline Espécies de aves & Espécies de insetos & Tipo de associação & Ocorrência \\
\hline $\begin{array}{l}\text { Picidae } \\
\quad \text { Colaptes campestris }\end{array}$ & Termitidae & Ninho em colônia terrícola & $3: 12(25)$ \\
$\begin{array}{l}\text { Psittacidae } \\
\text { Brotogeris chiriri }\end{array}$ & Nasutitermes spp. & Ninho em colônia arborícola & $3: 7(42,8)$ \\
$\begin{array}{l}\text { Brotogeris tirica } \\
\text { Strigidae }\end{array}$ & Nasutitermes spp. & Ninho em colônia arborícola & $2: 5(40)$ \\
$\quad$ Athene cunicularia & Cornitermes cf. silvestrii & Ninho em colônia terrícola & $1: 32(3,1)$ \\
$\begin{array}{l}\text { Trogonidae } \\
\text { Trogon surrucura }\end{array}$ & Nasutitermes spp. & Ninho em colônia arborícola & $4: 7(57,1)$ \\
Trogon viridis & Nasutitermes spp. & Ninho em colônia arborícola & $2: 3(66,6)$ \\
\hline
\end{tabular}

arborícolas ativos (BRIGHTSMITH, 2000; COLLAR, 2001). Na Amazônia peruana, $100 \%$ dos oito ninhos encontrados de Trogon melanurus Swainson, 1838, estavam em cupinzeiros ativos (BRIGHTSMITH, 2000). Por outro lado, a raridade de ninhos escavados em cupinzeiros arborícolas ativos, que encontramos para as duas espécies de Brotogeris Vigors, 1825 , não condiz com o estudo de BRIGHTSMITH (2000) na Amazônia peruana, que relata entre cerca de 80 a $90 \%$ dos 32 ninhos de duas espécies de Brotogeris em cupinzeiros ativos. Esta disparidade pode ser devida a preferências de nidificação entre as diferentes espécies destes psitacídeos em uma região e outra e, também, devido a diferenças relacionadas ao comportamento das espécies de cupins e à estrutura de seus ninhos nas duas regiões.

Nidificar em cupinzeiros terrestres ativos foi raro para uma espécie de Picidae e uma espécie de Strigidae (Tab. II). O pica-pau Colaptes campestris é conhecido pelo hábito de escavar seus ninhos em cupinzeiro terrícolas (Sick, 1997; WinkLer \& Christie, 2002; Dias et al., 2013) (Fig. 7), mas não encontramos informações sobre estes cupinzeiros conterem colônias ativas ou não. Sugerimos que os ornitólogos de campo, ao estudar reprodução de aves 


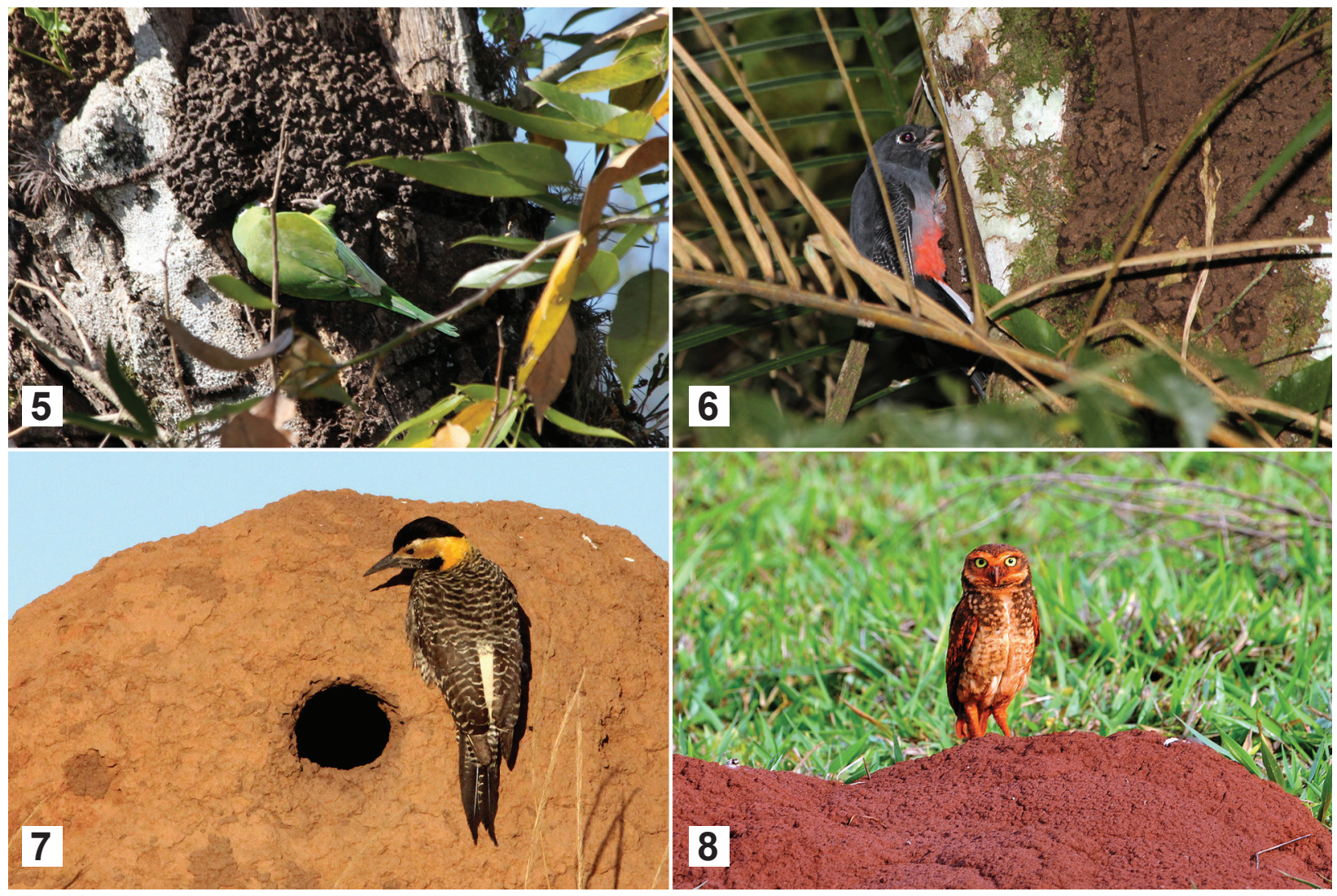

Figs 5-8. Associações de aves com Isoptera: 5, em árvore de local aberto, o periquito-de-encontro-amarelo (Brotogeris chiriri) inicia a escavação de ninho em uma colônia ativa de cupim arborícola (Nasutitermes species); 6, em árvore de mata, uma fêmea do surucuá-variado (Trogon surrucura) inicia a escavação de ninho em colônia ativa de cupim arborícola (Nasutitermes corniger), ao mesmo tempo que come alguns cupins; 7 , na entrada de seu ninho escavado em colônia de cupim terrícola (Cornitermes sp.) o pica-pau-do-campo (Colaptes campestris) espia os arredores antes de entrar; 8, no topo de uma colônia ativa de cupim terrícola (Cornitermes sp.) a coruja-buraqueira (Athene cunicularia) vigia os arredores. Créditos: Giulia B. D’Angelo (5), Ivan Sazima (6), Sávio F. Bruno (7), Camila Rocha (8).

que nidificam em cupinzeiros terrestres, tomem nota se o cupinzeiro está ativo ou não (veja PARANHOS et al., 2008). As corujas Athene cunicularia nidificam principalmente em tocas escavadas em barrancos ou no chão (SICK, 1997; KöNIG et al., 1999; MARKS et al., 1999). Aparentemente, nidificação em cupinzeiro terrestre ativo é uma ocorrência rara (presente trabalho) e necessita de confirmação por observações adicionais. O único ninho que achamos estava em cupinzeiro ativo de Cornitermes cf. silvestrii (Fig. 8), espécie cuja colônia por vezes tem formato variável, incluindo a de cogumelo, na qual a parte subterrânea é mais estreita que a epígea (VAlÉrio, 2006; Plaza et al. 2014). É possível, portanto, que a coruja tenha iniciado a escavação na parte larga e achatada do cupinzeiro e continuado na terra por fora da parte cilíndrica do cupinzeiro. De modo geral, informações sobre nidificação de aves em cupinzeiros terrestres ativos são escassas, uma das exceções sendo o trabalho de PARANHOS et al. (2008) sobre reprodução de Eupsittula aurea (Gmelin, 1788) no Sudeste do Brasil.

Das associações entre aves e insetos sociais, aqui relatadas, seguir formigas de correição é a mais conspícua e de ocorrência mais frequente (WILLIS \& ONIKI 1978, 2003; PARrinI, 2015). Acreditamos que em estudos deste tipo de associação surgiriam poucas novidades (e.g., SAZIMA,
2010). Por outro lado, em associações entre aves e ninhos de himenópteros deverão surgir exemplos adicionais, em particular de aves que nidificam próximo a colônias de formigas (MYERS, 1935; JANZEN, 1969), associação que não encontramos durante o presente estudo. Além disso, a riqueza de espécies de vespas também deverá aumentar, como recentemente demonstrado por MenEZEs et al. (2014). Já no caso de associações de aves com colônias de cupins, estudos adicionais poderão registrar riqueza de espécies de aves maior que a conhecida atualmente (BRIGHTSMITH, 2000; PARANHos et al., 2008; presente trabalho).

Amostras maiores poderão modificar as frequências de ocorrências aqui registradas, porém, a tendência possivelmente será mantida para a maioria das espécies. Entretanto, variações quali- e quantitativas regionais provavelmente ocorrem, o que justificaria este tipo de estudo em habitats e regiões distintas das aqui estudadas (veja recente trabalho de MenEzes et al., 2014). Estudos orientados para história natural deverão revelar maior riqueza de espécies e variedade de aves que se associam com colônias ativas de insetos sociais.

Agradecimentos. A Marlies Sazima e Micael E. Nagai pelo apoio constante; Micael também por sugestões ao manuscrito; Sidnei Mateus pelo apoio ao estudo de aves associadas a himenópteros e pela confirmação 
da identidade das vespas; ao editor de área e revisores anônimos pelas sugestões ao texto; CNPq por bolsa concedida anteriormente a IS.

\section{REFERÊNCIAS BIBLIOGRÁFICAS}

BRIGHTSMITH, D. J. 2000. Use of arboreal termitaria by nesting birds in the Peruvian Amazon. The Condor 102:529-538.

Cazal, S. R. A. L.; Azevedo Júnior., S. M.; Telino-Junior., W.; Neves, R. M. L.; Lira Filho, C. C. A.; Larrazábal, M. E. \& Branco, J. O. 2009. Biologia de Tolmomyias flaviventris (Wied, 1831) (Passeriformes, Tyrannidae) em Mata Atlântica, Pernambuco, Brasil. Ornithologia 3:67-72.

Collar, N. J. 1997. Family Psittacidae (parrots). In: DEL Hoyo, J.; ElLIOT, A. \& Sargatal, J. eds. Handbook of the birds of the world. Vol. 4. Sandgrouse to cuckoos. Barcelona, Lynx Edicions, p.280-477. 2001. Family Trogonidae (trogons). In: del Hoyo, J.; ElLIOT, A. \& Christie, D. eds. Handbook of the birds of the world. Vol.6. Mousebirds to hornbills. Barcelona, Lynx Edicions, p.80-127.

Dias, R. I.; Webster, M. S.; Goedert, D. \& Macedo, R. H. 2013. Cooperative breeding in the Campo Flicker I: breeding ecology and social behavior. The Condor 115:847-854

Eiten, G. 1970. A vegetação do Estado de São Paulo. Boletim do Instituto de Botânica (São Paulo) 7:1-147.

1992. Natural Brazilian vegetation types and their causes. Anais da Academia Brasileira de Ciências 64:35-65.

FitZPATRICK, J. W. 2004. Family Tyrannidae (tyrant-flycatchers). In: DEL Hoyo, J.; Elliot, A. \& Christie, D. eds. Handbook of the birds of the world. Vol. 9. Cotingas to pipits and wagtails. Barcelona: Lynx Edicions, p.170-462.

FraGa, R. M. 2011. Family Icteridae (New World blackbirds). In: DEL Hoyo, J.; Elliot, A. \& Sargatal, J. eds. Handbook of the birds of the world. Vol. 16. Tanagers to New World blackbirds. Barcelona, Lynx Edicions, p.684-807.

Goodenough, J.; McGuire, B. \& Jakob, E. 2010. Perspectives on animal behavior. 3ed. Hoboken, John Wiley \& Sons. 528p.

Haverschmidt, F. 1968. Birds of Suriname. Edinburgh, Oliver \& Boyd. 445 .

HiLty, S. L. 2011. Family Thraupidae (tanagers). In: Del Hoyo, J.; Elliot, A. \& Christie, D. eds. Handbook of the birds of the world. Vol. 16. Tanagers to New World blackbirds. Barcelona, Lynx Edicions, p.46-329.

JANZEN, D. H. 1969. Birds and the ant $\mathrm{x}$ acacia interaction in Central America, with notes on birds and other myrmecophytes. The Condor 71:240-256.

JoyCE, F. J. 1993. Nesting success of rufous-naped wrens (Campylorhynchus rufinucha) is greater near wasp nests. Behavioral Ecology and Sociobiology 32:71-77.

König, C.; WeICK, F. \& BeCKING, J. H. 1999. Owls: a guide to the owls of the world. New Haven, Yale University Press. 462p.

Marantz, C. A.; Aleixo, A.; Bevier, L. R. \& Patten, M. A. 2003. Dendrocolaptidae (woodcreepers). In: DEL Hoyo, J.; Elliot, A. \& Christie, D. eds. Handbook of the birds of the world. Vol. 8. Broadbills to tapaculos. Barcelona, Lynx Edicions, p. 358-447.

Marks, J. S.; Cunnings, R. J. \& Mikkola, H. 1999. Family Strigidae (typical owls). In: Del Hoyo, J.; Elliot, A. \& Sargatal, J. eds. Handbook of the birds of the world. Vol. 5. Barn-owls to humming birds. Barcelona, Lynx Edicions, p. 76-242.

Menezes, J. C. T.; Barbosa, B. C. \& Prezoto, F. 2014. Previously unreported nesting associations of the yellow-olive flycatcher (Tolmomyias sulphurescens) (Aves: Tyrannidae) with social wasps and bees. Ornitologia Neotropical 25:363-368.
Myers, J. G. 1935. Nesting associations of birds with social insects. Transactions of the Royal Entomological Society of London 83:11-23.

Paranhos, S. J.; Araújo, C. B. \& Machado, L. O. M. 2008. Comportamento reprodutivo de Aratinga aurea (Psittacidae) no sudoeste de Minas Gerais, Brasil. Revista Brasileira de Ornitologia 16:1-7.

PARRINI, R. 2015. Quatro estações, história natural das aves na Mata Atlântica: uma abordagem trófica. Rio de Janeiro, Technical Books. 354p.

Pizo, M. A. \& Melo, A. S. 2010. Attendance and co-occurrence of birds following army ants in the Atlantic rain forest. The Condor 112:571-578.

Plaza, T. G.; Carrijo, T. F. \& Cancello, E. M. 2014. Nest plasticity of Cornitermes silvestrii (Isoptera, Termitidae, Syntermitinae) in response to flood pulse in the Pantanal, Mato Grosso, Brazil. Revista Brasileira de Entomologia 58:66-70.

QuinN, J. L. \& Ueta, M. 2008. Protective nesting associations in birds. Ibis 150:146-167.

Robinson, S. K. 1985. Coloniality in the Yellow-rumped Cacique as a defense against nest predators. The Auk 102:506-519.

SAZIMA, I. 1989. Peach-fronted parakeet feeding on winged termites. Wilson Bulletin 101:656-657.

2010. Five instances of bird mimicry suggested for Neotropical birds: a brief reappraisal. Revista Brasileira de Ornitologia 18:328335.

SicK, H. 1997. Ornitologia brasileira. Rio de Janeiro, Nova Fronteira. $862 \mathrm{p}$.

SILVA, N. A. 2014. Tolmomyias sulphurescens (Spix, 1825). Wiki Aves - A Enciclopédia das Aves do Brasil. Disponível em $<$ http://www. wikiaves.com/1272727>. Acesso em 11.10.2014.

Somavilla, A.; Oliveira, M. L. \& Silveira, O. T. 2012. Guia de identificação dos ninhos de vespas sociais (Hymenoptera, Vespidae, Polistinae) na Reserva Ducke, Manaus, Amazonas, Brasil. Revista Brasileira de Entomologia 56:405-414.

Somavilla, A.; Fernandes, I. O.; Oliveira, M. L. \& Silveira, O. T. 2013. Association among wasps' colonies, ants and birds in Central Amazonian. Biota Neotropica 13:308-313.

Stotz, D. F. 1992. Buff-throated Saltator eats army ants. Wilson Bulletin 104:373-374.

SwarTZ, M. B. 2001. Bivouac checking, a novel behavior distinguishing obligate from opportunistic species of army-ant-following birds. The Condor 103:629-633.

VALÉRIO, J. R. 2006. Cupins-de-montículo em pastagens. Documentos 160. Campo Grande, Embrapa Gado de Corte. 35p.

Willis, E. O. \& ONIKI, Y. 1978. Birds and army ants. Annual Review of Ecology and Systematics 9:243-263.

2003. Aves do estado de São Paulo. Rio Claro, Editora Divisa. $398 \mathrm{p}$.

2008. Aves seguidoras de correições de formigas nas Américas e África. Revista ACOALFAplp: Acolhendo a Alfabetização nos Países de Língua portuguesa 2. Disponível em $<$ http://dx.doi. org/10.11606/issn.1980-7686.v2i4p301-320>. Acesso em 29.10.2014.

Winkler, H. \& Christie, D. A. 2002. Family Picidae (woodpeckers). In: Del Hoyo, J.; Elliot, A. \& SARgatal, J. eds. Handbook of the birds of the world. Vol. 7. Jacamars to woodpeckers. Barcelona, Lynx Edicions. p.296-558.

ZimMer, K. J. \& IsLER, M. L. 2003. Thamnophilidae (typical antbirds). In: DEL Hoyo, J.; Elliot, A. \& Christie, D. eds. Handbook of the birds of the world. Vol. 8. Broadbills to tapaculos. Barcelona: Lynx Edicions, p.448-681. 